\title{
Breast cancer incidence and mortality in a transitioning Chinese population: current and future trends
}

\author{
I O L Wong*,1, C M Schooling ${ }^{1,2}$, B J Cowling ${ }^{1}$ and G M Leung ${ }^{1}$ \\ ${ }^{1}$ School of Public Health, Li Ka Shing Faculty of Medicine, The University of Hong Kong, Hong Kong SAR, China and ${ }^{2}$ City \\ University of New York School of Public Health and Hunter College, New York, NY, USA
}

Background: Projections of future trends in cancer incidence and mortality are important for public health planning.

Methods: By using 1976-2010 data in Hong Kong, we fitted Poisson age-period-cohort models and made projections for future breast cancer incidence and mortality to 2025 .

Results: Age-standardised breast cancer incidence (/mortality) is projected to increase (/decline) from 56.7 (/9.3) in 2011-2015 to 62.5 (/8.6) per 100000 women in 2021-2025.

Conclusions: The incidence pattern may relate to Hong Kong's socio-economic developmental history, while falling mortality trends are, most likely, due to improvements in survival from treatment advancement and improved health service delivery.

Breast cancer is the most common malignancy and the leading cause of cancer-related death in women, accounting for $10 \%$ of new malignancies worldwide annually, and $\sim 22 \%$ of malignancies among women (Ferlay et al, 2004). In Hong Kong (HK) the most westernised and urbanised city in China, breast cancer is the most common cancer and the third leading cause of cancer-related mortality among women (Cancer Statistics, 2013). There are clear differences between populations in the East and the West in terms of breast cancer incidence and mortality (Figure 1), the precise aetiology of which remains unclear.

Age-period-cohort (APC) models allowing examination of trends in disease rates over time (Holford, 1991, 1992) have long been deployed to further understanding of the biologic and environmental causes of cancer (Holford, 1991, 1992). By using APC methods, we estimated the relative contribution of effects from age, period and birth cohort to trends in breast cancer incidence and mortality, and projected future trends to the medium term.

\section{MATERIALS AND METHODS}

We obtained age-specific breast cancer incidence, death data (defined according to the International Classification of Diseases
(ICD) codes as ICD-8 174, ICD-9 174 and ICD-10 C50, C50.0C50.9) and mid-year population figures for the years 1976-2010 from the HK Cancer Registry (2013), the Death Registry and the Census and Statistics Department, respectively. We calculated the age-standardised incidence and mortality rates in $\mathrm{HK}$ according to the World Standard Population in 2000. Non-linearities in trends of varying time periods were characterised using segmented annual percent change from segmented regression (Clegg et al, 2009) or join-point regression analysis (Kim et al, 2000).

We modelled breast cancer incidence and mortality using APC models (details in Supplementary Information 1) (Clayton and Schifflers, 1987; Holford, 1991, 1992). We grouped the mortality/ incidence and population data into fourteen 5-year age groups from $20-24$ years to 85 or above, and seven 5 -year periods from 1976-1980 to 2006-2010. This classification resulted in 20 birth cohorts centred at 5-year intervals beginning in the 1890s. The second and the penultimate periods and the central birth cohort were defined as reference groups to generate identifiable estimates. We used Bayesian inference to estimate the model parameters (Bray et al, 2000; Bray, 2002; Brennan and Bray, 2002; Baker and Bray, 2005). We used the resulting models to project agestandardised breast cancer incidence and mortality rates to medium term (until 2021-2025), with 95\% projection intervals.

*Correspondence: IOL Wong; E-mail: iolwong@hku.hk

Received 9 June 2014; revised 4 September 2014; accepted 9 September 2014; published online 7 October 2014

(c) 2015 Cancer Research UK. All rights reserved 0007-0920/15 


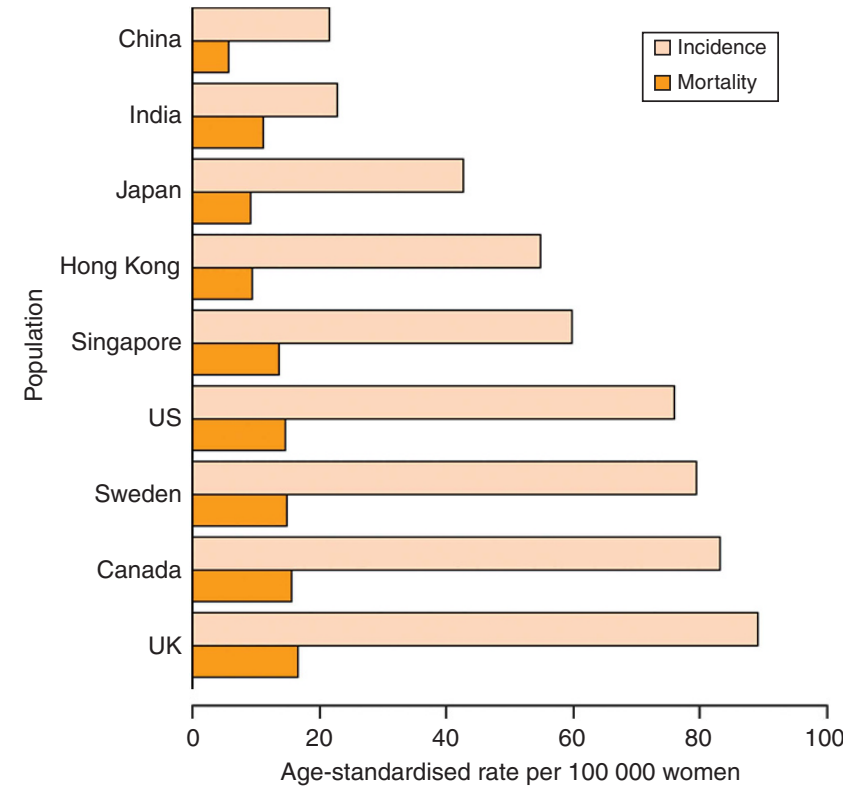

Figure 1. Age-standardised rates of female breast cancer incidence and mortality for women in 2008 (Data source: GLOBCAN 2008).

\section{RESULTS}

The trends in age-standardised breast cancer incidence and mortality rates by 5 -year periods from 1976 and 2010 are shown in Figure 2 and Supplementary Table 1. The fourth period centred at 1993 was marked by a join-point at which the slope changed significantly for incidence and mortality trends in 1976-2010.

The estimated parameters for the age, period and cohort components for incidence (upper panels) and mortality (lower panels) models are shown in Figure 3. Due to the well-known identifiability problem of APC models, where the effects of the three components are linearly dependent, only second-order changes (i.e., inflection points) are interpretable. Figure 3 shows clear second-order changes are visible for both the age effects and the cohort effects for incidence and mortality with negligible second-order changes in period effects. Downward inflections in the age-specific breast cancer rates occurred at around 45-50 years for incidence and 65-70 years for mortality. The first two inflection points for the cohort curves have similar downturns around 1910 and upturns around 1930 for both incidence and mortality, but the third inflection points differ, with a downturn around 1960 for incidence and around 1950 for mortality.

Age-standardised incidence rate rose on average $1.69 \%$ per annum in the three decades between 1976 and 2010. On the basis of these trends, we predicted that age-standardised breast cancer incidence rates would increase from 56.7 cases in 2011-2015 to 62.5 per 100000 women in 2021-2025. In contrast, age-standardised mortality rates decreased on average $0.02 \%$ per year between 1976 and 2010. The rate is projected to decline from 9.3 deaths in 2011-2015 to 8.6 deaths per 100000 women in 2021-2025.

Disparities in the disease rates by age group were observed (Supplementary Figure 1). Incidence is projected to increase into the near future for women $\geqslant 55$ years, while mortality is projected to decline for women aged $<65$ years but increase for women aged $\geqslant 65$ years.

\section{DISCUSSION}

We predict that age-standardised breast cancer incidence in $\mathrm{HK}$ will continue to increase at a rate of $0.65 \%$ per annum, while

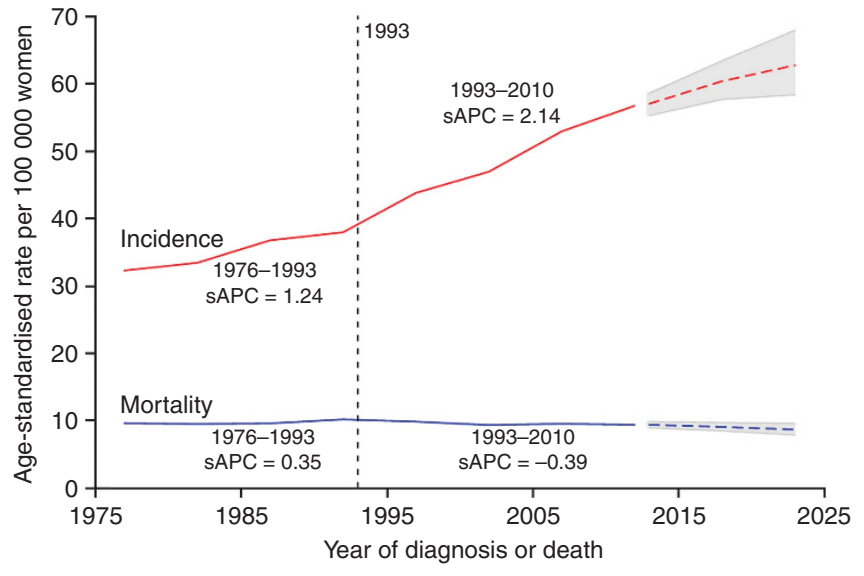

Figure 2. Annual age-standardised female breast cancer incidence (red line) and mortality (blue line) rates in Hong Kong from 1976 to 2010 and projected incidence and mortality (dotted lines) to 2025 with $95 \%$ credible intervals (grey area). Notes: The sAPC (annual percent change from segmented analysis) is significantly different from zero $(P<0.05)$, from which the period centred at 1993 (vertical dotted line) was marked by a join-point at which the slope changed significantly in both the incidence and mortality trends.

age-standardised mortality will decrease at $0.56 \%$ per annum over the next 15 years. Cumulatively, this represents a projected increase of $10.2 \%$ in incidence and a decrease of $7.5 \%$ in mortality rates during 2010-2025. Similar to our previous findings (Wong et al, 2007), the rising incidence trends seem to have been driven mainly by ageing and cohort effects, with no clear trends by calendar time period. On the other hand, we observed that the overall mortality trends appeared to be relatively stagnant over the last three decades. Given that HK does not operate a mass mammographic screening and only the low levels of mammography screening in our population occur (Leung et al, 2008), these trends could be most likely attributed to improvements in survival including advances in treatment care such as use of Paclitaxel from the 1990s and adjuvant hormonal (Early Breast Cancer Trialists' Collaborative Group, 2005; Gibson et al, 2009; Burstein et al, 2010; Cuzick et al, 2010) from the 1970s and targeted immunotherapy (Slamon et al, 2001; Madarnas et al, 2008; Costa et $a l, 2010)$ in the 2000s, or a different case-mix. We expect mortality to continue to decrease in the future. These findings might also serve as a reliable epidemiological sentinel for the rest of developing China.

Similar notable birth cohort effects occurred for both breast cancer incidence and mortality in the first half of the 20th century. Here, we attempt to interpret the cohort-driven trends and to generate hypotheses based on the relevance of social history in the evolution of population disease risk, in addition to from the perspective of well-known contemporaneous determinants such as lifestyle factors. Deceleration for the 1910s birth cohorts may be an artefact of HK's migration history. Acceleration for the 1930s birth cohorts coincided with the first cohort of women who by migrating from China to HK in 1945-1955 were the first generation to reach maturity in comparatively developed HK, where gross domestic product per head in 1951 was about five times higher than in China (Maddison, 2001). The deceleration for the birth cohorts a generation later around 1960 could represent an upper threshold in the population effects of transitioning to a developed society for East Asians, despite lower fertility, delayed childbearing (Herrinton et al, 1994; Liao et al, 2003) (Supplementary Figure 2) and increased oral contraceptive use for women born after 1960. On the other hand, migration studies suggest it takes several generations for the full effects of economic transition to become 

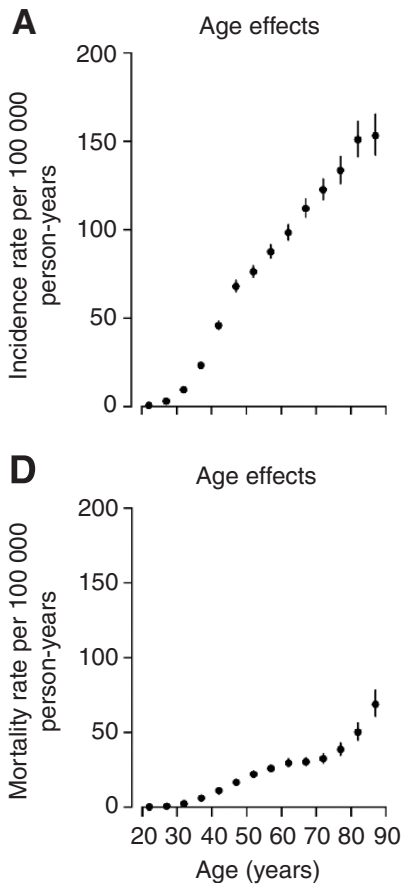
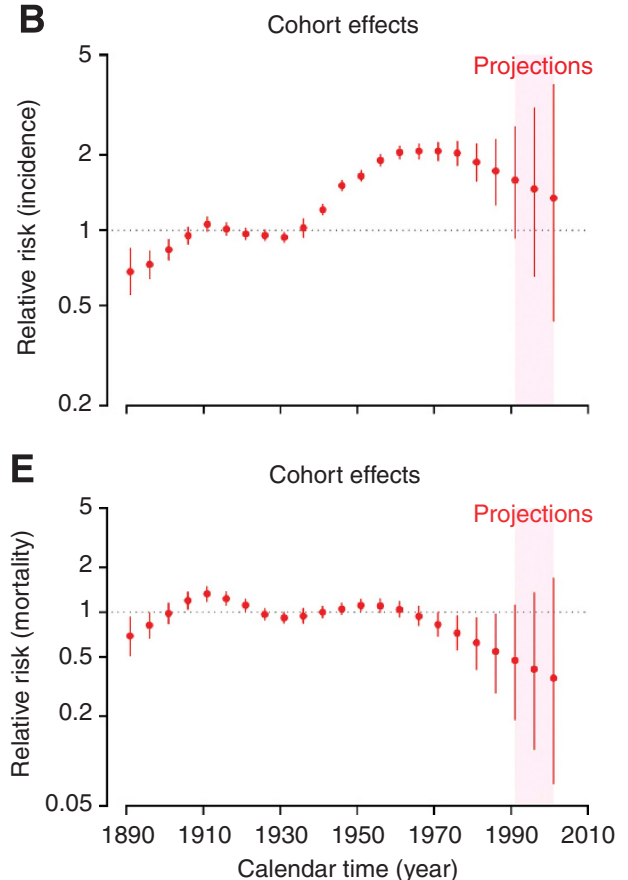
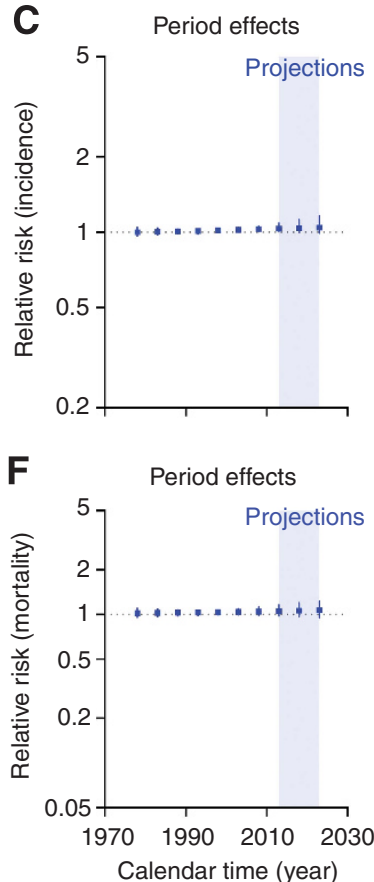

Figure 3. Parameter estimates of age (black), period (blue) and cohort (red) effects from two age-period-cohort models for female incidence (upper panels) and mortality (lower panels) trends due to breast cancer (DIC $=916.60$ for incidence rate model; $\mathrm{DIC}=737.44 \mathrm{for}$ mortality rate model) with projections (shaded area). Leftmost upper panel (A): Estimated age-specific annual female incidence rates due to breast cancer in 5year age groups in Hong Kong with 95\% credible intervals. Middle and rightmost upper panel (B, C): Estimated relative risks for 10-year birth cohort (beginning in the calendar year 1889) and 5-year calendar period (beginning from 1976) effects associated with the incidence cases with 95\% credible intervals, including projected cohort effects for birth cohorts centred on the years 1993, 1998 and 2003 (shaded areas) and projected period effects for periods centred on the years 2013, 2018, 2023 (shaded area). Leftmost lower panel (D): Estimated age-specific annual female mortality rates due to breast cancer in 5-year age groups in Hong Kong with $95 \%$ credible intervals. Middle and rightmost lower panels (E, F): Estimated relative risks for 10-year birth cohort (beginning in the calendar year 1889) and 5-year calendar period (beginning from 1976) effects associated with the deaths with 95\% credible intervals, including projected cohort effects for birth cohorts centred on the years 1993,1998 and 2003 (shaded area) and projected period effects for periods centred on the years 2013, 2018, 2023 (shaded area).

evident for female hormonal-related cancers (Herrinton et al, 1994; Liao et al, 2003). Notably, the mortality deceleration started earlier for generations born around 1950s, perhaps due to generational differences in awareness and inclination to seek screening and treatment. Conversely, if survival remains high for these women as they get older, then this would point to the existence of an unidentified protective factor which has counteracted the mortality associated with changing socio-economic and demographic patterns.

With respect to the age curves, downward inflection points in incidence and mortality rates were around $45-50$ years and $65-70$ years, respectively. The downward inflection in incidence at the age of menopause coincides with Clemmesen's hook, which is classically recognised in developed countries (Clemmesen, 1948). The subsequent downward inflection in mortality may represent the lag between incidence and mortality.

In most Caucasian populations in the developed West, increases in breast cancer incidence have mainly occurred in women aged $\geq 50$ years, whereas reductions have been observed in those aged 35-49 years (Hery et al, 2008). We observed similar patterns, although only sporadic, opportunistic mammography screening occurs with relatively low and socially patterned coverage in HK (Leung et al, 2008).

We also projected a reduction in age-specific breast cancer mortality rates for women aged $<65$ years, and a rising trend in the rates among women $\geqslant 65$ years in the next 15 years. When comparing our trends with western countries, a reduction in mortality trends has been observed in the younger age group $(<50$ years) in countries where screening of young women is rare (Denmark, Finland, Netherlands, Norway, UK) but also in those where screening is common (France, Germany, Sweden) (Autier et al, 2010). The relatively larger reduction in younger women involved may reflect more effective cancer treatment and better response to treatment (Autier et al, 2010). Similarly, the breast cancer deaths in United States and United Kingdom decreased at ages 20-69 years in the recent decade, probably due to many factors including increased use of hormonal and cytotoxic treatments for both early and advanced disease and increased awareness of breast cancer (Peto and Boreham, 2000). These same factors could apply in the $\mathrm{HK}$ context. Increased mortality projections for older women could be due to less frequent diagnostic activity, less intensive treatment and more frequent diagnosis of cancer at advanced stages resulting in poorer survival (Eaker et al, 2006; Autier et al, 2010). Investigation of the underlying reasons is needed.

There are two potential limitations in this study. First, APC analyses are descriptive in nature and we can only speculate the aetiologies of the changes observed. Second, we cannot quantify the survival benefit due to progress in early detection and treatment care in reducing disease risk in past trends, due to lack of relevant survival data in our Chinese population. We however estimated the complement of the mortality to incidence ratio $(1-(M / I)$ ratio $)$ as a proxy indicator of 5-year relative survival of breast cancer (Supplementary Figure 3) (Asadzadeh Vostakolaei et al, 2011). The proxy shows improved survival from breast cancer over times, as the curve of $1-(M / I)$ ratio appears to be increasing. 


\section{ACKNOWLEDGEMENTS}

We thank the Census and Statistics Department of the Government of the Hong Kong Special Administrative Region for their support and for facilitating data access, although they have no role in the design, analysis, interpretation or decision to submit for publication. We thank Patrick CN Wong for his support in data retrieval and literature review. This project was supported by the Health and Medical Research Fund; Food and Health Bureau; Government of the HK Special Administrative Region, China (Grant number: 10111031).

\section{REFERENCES}

Asadzadeh Vostakolaei F, Karim-Kos HE, Janssen-Heijnen ML, Visser O, Verbeek AL, Kiemeney LA (2011) The validity of the mortality to incidence ratio as a proxy for site-specific cancer survival. Eur J Public Health 21(5): 573-577.

Autier P, Boniol M, LaVecchia C, Vatten L, Gavin A, Héry C, Heanue M (2010) Disparities in breast cancer mortality trends between 30 European countries: restrospective trend analysis of WHO mortality database. $\mathrm{Br}$ Med J 341: c3620.

Baker A, Bray I (2005) Bayesian projections: what are the effects of excluding data from younger age groups? Am J Epidemiol 162(8): 798-805.

Bray I (2002) Application of Markov chain Monte Carlo methods to projecting cancer incidence and mortality. Appl Stat 51: 151-164.

Bray I, Brennan P, Boffetta P (2000) Projections of alcohol- and tobaccorelated cancer mortality in Central Europe. Int J Cancer 87(1): 122-128.

Brennan P, Bray I (2002) Recent trends and future directions for lung cancer mortality in Europe. Br J Cancer 87(1): 43-48.

Burstein HJ, Prestrud AA, Seidenfeld J, Anderson H, Buchholz TA, Davidson NE, Gelmon KE, Giordano SH, Hudis CA, Malin J, Mamounas EP, Rowden D, Solky AJ, Sowers MR, Stearns V, Winer EP, Somerfield MR, Griggs JJ (2010) American Society of Clinical Oncology clinical practice guideline: update on adjuvant endocrine therapy for women with hormone receptor-positive breast cancer. J Clin Oncol 28(23): 3784-3796.

Cancer Statistics (2013) http://www3.ha.org.hk/cancereg/e_alb.asp. Accessed November 2013.

Clayton D, Schifflers E (1987) Models for temporal variation in cancer rates, II: age-period-cohort models. Stat Med 6: 469-481.

Clegg LX, Hankey BF, Tiwari R, Feuer EJ, Edwards BK (2009) Estimating average annual per cent change in trend analysis. Stat Med $\mathbf{2 8}$ 3670-3682.

Clemmesen J (1948) Carcinoma of the breast. Br J Radiol 21: 583-590.

Costa RB, Kurra G, Greenberg L, Geyer CE (2010) Efficacy and cardiac safety of adjuvant trastuzumab-based chemotherapy regimens for HER2-positive early breast cancer. Ann Oncol 21: 2153-2160.

Cuzick J, Sestak I, Baum M, Buzdar A, Howell A, Dowsett M, Forbes JF (2010) Effect of anastrozole and tamoxifen as adjuvant treatment for early-stage breast cancer: 10-year analysis of the ATAC trial. Lancet Oncol 11(12): $1135-1141$.

Eaker S, Dickman PW, Bergkvist L, Holmberg L. The Uppsala/Orebro Breast Cancer Group (2006) Differences in management of older women influence breast cancer survival: results from a population-based database in Sweden. PLoS Med 3(3): e25.
Early Breast Cancer Trialists' Collaborative Group (2005) Effects of chemotherapy and hormonal therapy for early breast cancer on recurrence and 15-year survival: an overview of the randomised trials. Lancet 365 : $1687-1717$.

Ferlay J, Bray F, Pisani P, Parkin DM. GLOBOCAN (2002) Cancer Incidence, Mortality and Prevalence Worldwide. IARC CancerBase No. 5, version 2.0, IARC Press: Lyon (2004).

Gelman A, Rubin DB (1992) Inference from iterative simulation using multiple sequences. Stat Sci 7(4): 457-511.

Gibson L, Lawrence D, Dawson C, Bliss J (2009) Aromatase inhibitors for treatment of advanced breast cancer in postmenopausal women. Cochrane Database Syst Rev 4: CD003370.

Herrinton LJ, Standford JL, Schwartz SM, Noel SW (1994) Ovarian cancer incidence among Asian migrants to the United States and their descendants. J Natl Cancer Inst 86: 1336-1339.

Hery C, Ferlay J, Boniol M, Autier P (2008) Quantification of changes in breast cancer incidence and mortality since 1990 in 35 countries with Caucasian-majority populations. Ann Oncol 19: 1187-1194.

Holford T (1991) Understanding the effects of age, period and cohort on incidence and mortality rates. Annu Rev Public Health 12: 425-457.

Holford T (1992) Analyzing the effects of age, period and cohort on incidence and mortality rates. Stat Methods Med Res 1: 317-337.

Hong Kong Cancer Registry (2013) Hong Kong Cancer Registry http://www3.ha.org.hk/cancereg/faq.html. Accessed November 2013.

Kim HJ, Fay MP, Feuer EJ, Midthune DN (2000) Permutation tests for joinpoint regression with applications to cancer rates. Stat Med 19: 335-351(correction: 2001;2020:2655).

Leung GM, Woo PPS, Cowling BJ, Tsang CS, Cheung AN, Ngan HY, Galbraith K, Lam TH (2008) Who receives, benefits from and is harmed by cervical and breast cancer screening among Hong Kong Chinese. J Public Health. 30: 282-292.

Liao CK, Rosenblatt KA, Schwartz SM, Weiss NS (2003) Endometrial cancer in Asian migrants to the United States and their descendants. Cancer Causes Control 14: 357-360.

Madarnas Y, Trudeau M, Franek JA, McCready D, Pritchard KI, Messersmith H (2008) Adjuvant / neoadjuvant trastuzumab therapy in women with HER-2/neu-overexpressing breast cancer: a systematic review. Cancer Treat Rev 34: 539-557.

Maddison A (2001) The World Economy. A Millennial Perspective. Organisation for Economic Co-operation and Development, Development Centre: Paris, France.

Peto R, Boreham J (2000) UK and USA breast cancer deaths down 25\% in year 2000 at ages 20-69 years. Lancet 355: 1822.

Slamon DJ, Leyland-Jones B, Shak S, Fuchs H, Paton V, Bajamonde A, Fleming T, Eiermann W, Wolter J, Pegram M, Baselga J, Norton L (2001) Use of chemotherapy plus a monoclonal antibody against HER2 for metastatic breast cancer that overexpresses HER2. N Engl J Med 344(11): 783-792.

Spiegelhalter DJ, Best NG, Carlin BP, van der Linde A (2002) Bayesian measures of model complexity and fit. J R Stat Soc B 64: 583-639.

Wong IOL, Cowling BJ, Schooling CM, Leung GM (2007) Age-period-cohort projections of breast cancer incidence in a rapidly transitioning Chinese population. Int J Cancer 121: 1556-1563.

This work is published under the standard license to publish agreement. After 12 months the work will become freely available and the license terms will switch to a Creative Commons AttributionNonCommercial-Share Alike 3.0 Unported License.

Supplementary Information accompanies this paper on British Journal of Cancer website (http://www.nature.com/bjc) 